\title{
Cloud based multi-agent architecture for effective planning and scheduling of distributed manufacturing
}

\author{
Nishikant Mishra ${ }^{1}$, Akshit Singh $^{2}$, Sushma Kumari ${ }^{3}$ and Kannan Govindan ${ }^{4}$ \\ ${ }^{1}$ Norwich Business School, University of East Anglia, Norwich, UK, N.mishra@uea.ac.uk \\ ${ }^{2}$ Norwich Business School, University of East Anglia, Norwich, UK, Akshit.Singh@uea.ac.uk \\ ${ }^{3}$ Sussex Business School, University of Sussex, Sussex, UK, sk546@sussex.ac.uk \\ ${ }^{4}$ Center for Engineering Operations Management, Department of Technology and Innovation, University of \\ Southern Denmark, Odense, Denmark, gov@sam.sdu.dk
}




\begin{abstract}
In modern world, manufacturing processes have become very complex because of consistently fluctuating demand of customers. Numerous production facilities located at various geographical locations are being utilized to address the demands of their multiple clients. Often, the components manufactured at distinct locations are being assembled in a plant to develop the final product. In this complex scenario, manufacturing firms have to be responsive enough to cope with the fluctuating demand of customers. To accomplish it, there is need to develop an integrated, dynamic, autonomous system. In this article, a self-reactive cloud based multi agent architecture for distributed manufacturing system is developed. The proposed architecture will assist manufacturing industry to establish real time information exchange between the autonomous agents, clients, suppliers and manufacturing unit. The mechanism described in this study demonstrates how the autonomous agents interact with each other to rectify the internal discrepancies in manufacturing system. It can also address the external interferences like variations in client's orders to maximise the profit of manufacturing firm in both short and long term. Execution process of proposed architecture is demonstrated using simulated case study.
\end{abstract}

Keywords: multi agent architecture, cloud-computing technology, distributed manufacturing.

\title{
1. Introduction
}

Now a day, manufacturing firms utilizes the concept of distributed manufacturing. They have manufacturing plants at various geographical locations producing different products, which are later on assembled to develop the final product. It helps to boost the efficiency of manufacturing firms. Consequently, a particular manufacturing firm has multiple clients with varying profit margin associated with each of them. However, coordination among the fragmented manufacturing units is mandatory to cope with the fluctuating demand of customers.

In the past, the performance attribute of a manufacturing firm was defined by enhancing the sales and cutting down the price. Qualitative aspects like customer's choice and performance in delivering goods replaced these traits in 1980s. However, since then, global market has become customer driven. Therefore, an uncertainty factor is now being associated with the demand patterns of the market. Manufacturing firms have to be highly agile and responsive to keep up the customer satisfaction in order to sustain in modern economy. Therefore, the current performance indicator of a manufacturing firm is its rapid responsiveness to the volatile demand patterns of market. Apart from external factors, they also have to address the internal uncertainties like breakdown of machines, supply of raw materials, etc. All these factors collectively play a crucial role in framing the modern manufacturing strategies. 
Traditionally, the manufacturing strategies were developed to keep the lead times, cost, and inventory level in check. As the trend in manufacturing firms are now more aligned towards responsiveness, the traditional manufacturing firms are unable to cope with varying demand patterns of modern market. The modern manufacturing strategies must be framed to boost the ability to reconfigure, scalability, agility, ability to restructure and reusability. These things will in turn increase the manufacturing efficiency and product quality. These strategies will assist in turn to raise the capability to adapt, flexibility in addressing the internal issues of manufacturing firms like machine failure, machine unavailability, etc.

The responsiveness and agility in a manufacturing firm can be accomplished by effectively interlinking the manufacturing scheduling and process planning. It will enable them to cope with the external factors like frequent variations in demand patterns and the internal factors like machine unavailability, machine breakdown, bottlenecks in production, etc.

This study proposes cloud based multi agent architecture for boosting the efficiency and revenue of distributed manufacturing firms. It facilitates the efficient integration of manufacturing scheduling and process planning. This framework helps to simultaneously optimize manufacturing scheduling and process planning to develop the most appropriate manufacturing plan with respect to the fluctuating demand patterns of the market. A cloud based multi agent system brings the different units of manufacturing firm and the clients associated with them on a single platform. This framework comprises of eleven autonomous agents, which are capable of decision-making at different stages with minimum human interference. Two of the agents (planning agent and negotiation agent) are playing crucial role in this study. They are utilizing a very sophisticated tool called algorithm portfolio. The execution of this cloud based multi agent framework is demonstrated in simulated case study in section 4. In the proposed architecture, we have demonstrated how different agents will interact with each other to overcome both internal and external discrepancies with less human interventions. It also illustrates how manufacturing firm will design strategy to address the external interferences like variations in client's orders to maximise the profit of manufacturing firm in both short and long term.

The organization of this article is as follows: Section 2 consists of literature review. Section 3 describes multi agent framework. Section 4 is composed of case study. The conclusion of this article is given in section 5 .

\section{Literature Review}

The modern manufacturing industry has to employ different techniques of distributed manufacturing to address the needs of consumer driven global market. The literature is full of extensive research on integration methodologies like multi agent simulation, game theory, Nash equilibrium, genetic algorithm (He et al., 2014; Jose et al., 2015; Wang et al., 2003; Wang et al., 2001). Various approaches for integrated process planning and production scheduling have been proposed such as just-in-time process planning, flexible process 
planning, dynamic process planning, non-linear process planning (Chen, 2010); Huang et al., 2013; Kim et al., 2009).

Multi agent system (MAS) assists in active communication in distributed manufacturing. MAS concept is derived from Distributed and Artificial Intelligence (DAI). Agents work together to find solution of sophisticated issues and problems with accuracy with less human intervention. Different researchers have utilized agent concept in finding solution to planning, design and scheduling problems (Li et al., 2010; Jose et al., 2015). MAS have certain characteristics like autonomy, reactive, proactive and social interaction (Moyaux and Chaibdraa, 2006; Swaminathan et al., 1998). They have wide applications in manufacturing industry like integration, interaction, negotiation, demand forecasting and efficient planning and scheduling (Caridi and Cavalieri, 2004; Bo and Zhiming, 2003;).

Yu et al. (2015) has proposed a negotiation protocol between multiple material supplier agent (MSAs) and multiple manufacturer agent (MAs). It will help to determine the final allocation scheme to optimise the aggregate profit of entire network of supply chains. Chien and Tien (2013) have suggested a negotiation framework for optimum utilisation of design resources in distributed product development. Yu and Wong (2015) have proposed a multi agent framework to do the supplier selection of multiple products by taking into account the synergy among the products. Lim et al. (2013) has worked on integrating production scheduling and process planning of multi-site manufacturing facilities by utilising multi agent system to develop the most economical and efficient manufacturing plan to address the demand. Wong and Fang (2010) have presented a unique multi agent protocol called Extended Contract-Net-like multilateral Protocol (ECNPro) for addressing buyer seller negotiations within a supply chain. Huang and Liao (2012) have suggested a distributed parallel machine scheduling for electro etching process of manufacturing of aluminium foil. Renna (2011) has presented a coordinated concept for multi agent framework as per the calculation of internal and external indexes of generic manufacturing cell.

Cloud Computing Technology (CCT) is an easy to implement method. Some of the major advantages of CCT are cost reduction in hardware, processing, availability of real time information, faster deployment, enhanced flexibility and agility and reduced IT support staff (Angela et al, 2012; Guo et al, 2014). CCT has wide applications in automobile sector, banking, logistics, education, and healthcare. Figure 1 shows three different cloud deployment models, which are public, private and hybrid cloud (Al-Hudhaif and Alkubeyyer, 2011; Singh et al, 2015).

In the past, researchers have developed various methods to resolve the complexities and faults occurring in the manufacturing firms. Almost all of these methods and techniques addressed the internal and external interferences of manufacturing firms separately. However, both of these complexities (internal and external) are interlinked. Only few researchers have developed robust methods to tackle with both kinds of faults simultaneously. These kinds of modern procedures need an automated system, which can bring all the stakeholders of manufacturing companies on a single platform with minimum human interference. Keeping this in mind, an automated mechanism is proposed in this article, which will bring multiple 
stakeholders of manufacturing firms on the same platform and is capable to efficiently address both the internal and external disruptions in the manufacturing system processes.

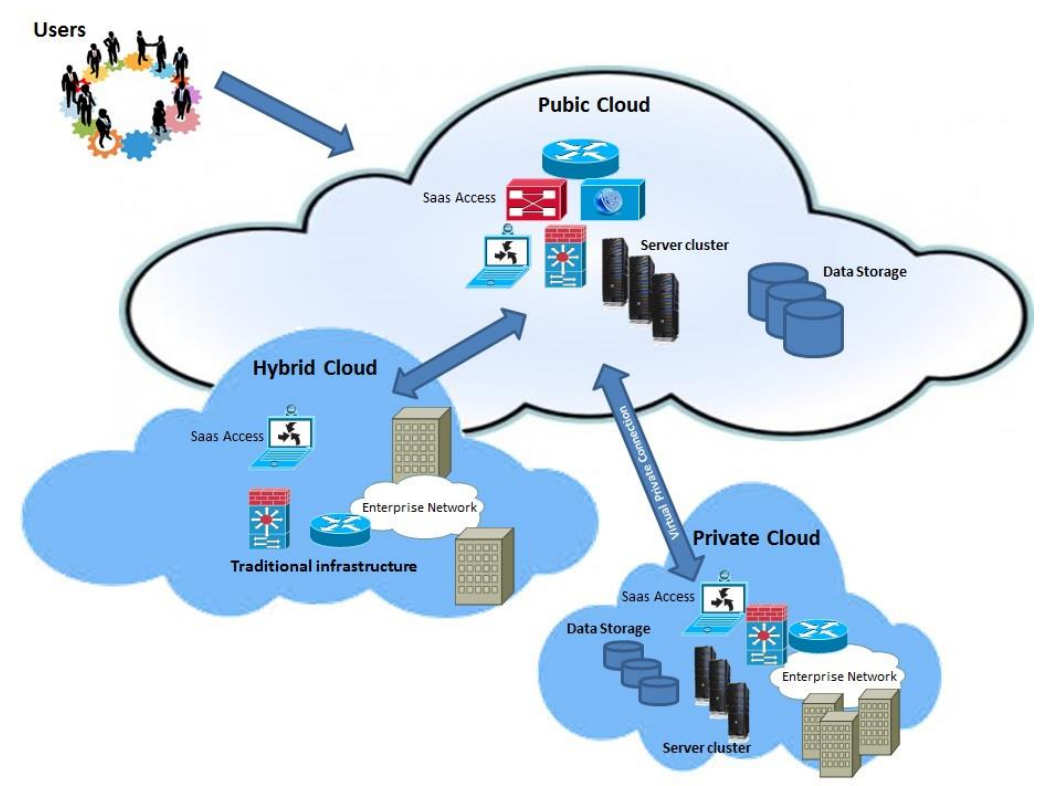

Figure 1. Deployment models of cloud computing technology

This article proposes cloud based multi agent architecture for distributed manufacturing system. The beauty of this framework is its capability to update real time process planning and scheduling decisions in the scenario of fluctuating consumer demand. The eleven autonomous agents utilised in this framework, coordinate with each other to address the internal and external interferences. They can update their manufacturing plan accordingly to maximise the short and long-term profit of the manufacturing firm.

\section{Multi-agent Framework}

The schematic diagram of proposed multi agent architecture is being shown in figure 2 . It consists of purchase order collection agent, supplier selection agent, planning agent, material planning agent, transport agent, administrative agent, forecasting agent, maintenance agent, knowledge base agent and design agent. These agents actively coordinate with each other throughout the manufacturing process. The characteristic of the above mentioned agents are being described as following: 


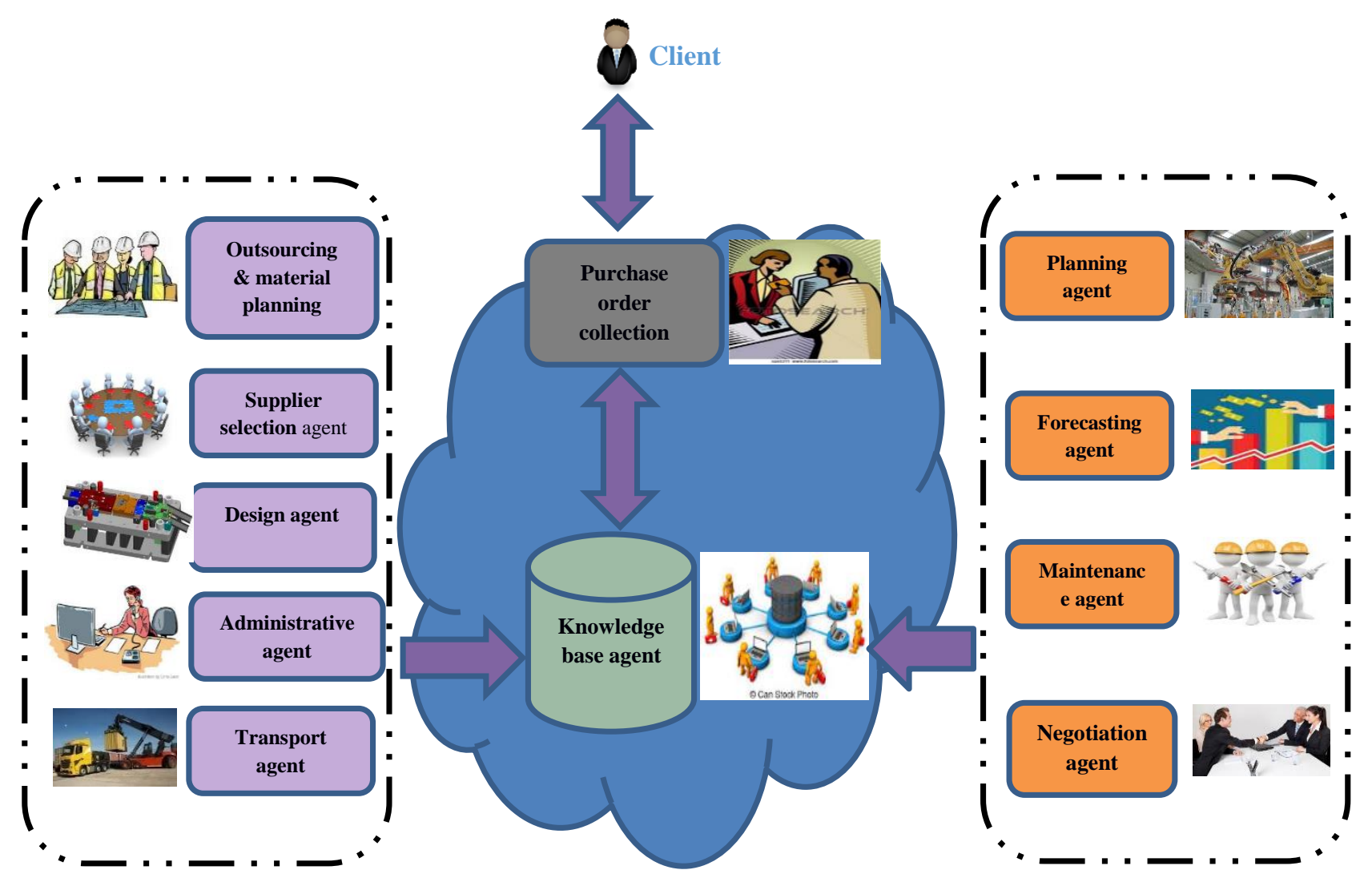

Figure 2 Cloud based multi-agent architecture for distributed manufacturing

3.1 Purchase order collection agent - It will obtain the orders from the clients in lieu of their manufacturing plants. They will interact with planning agent to decide the due date for delivery of ordered products. Then, the payment method will be decided. It could be via online banking, cash, wire transfer, cheque, bank draft or paying in instalments as per their interaction with administrative agent. The mode of delivery of ordered products is being decided with consent of transport agent, whether it will be rail freight, road transport using lorries or airfreight. Purchase order collection agent keeps updating knowledge base agent throughout its activities. It also has the additional ability to update itself online as well as offline.

3.2 Material planning agent - It operates in collaboration with forecasting agent and planning agent. This agent also performs the record keeping for availability of raw material in the manufacturing plant. When it receives the information from forecasting agent about demand in future, it will calculate bill of material. This information is being 
sent to supplier selection agent to find out the appropriate supplier to buy these raw materials. Then, it decides whether a particular operation has to be performed within the manufacturing plant or it has to be outsourced to some external industrial partner. This phenomenon depends on the due date of delivery of final manufactured products to clients, available resources within the manufacturing plant and expectations of the client. When material-planning agent realizes that a particular operation has to be outsourced, it will contact supplier selection agent to find the appropriate outsourcing partner. In the proposed architecture, cloud-computing technology will increase the information visibility in supply chain. Supplier can see inventory information of manufacturer. It will help supplier to develop an effective plan for meeting future demands and to minimise the inventory and lead-time.

3.3 Supplier selection agent -As soon as supplier selection agent receives the instructions from material planning agent, it starts to look for the appropriate supplier for raw material or external industrial partner who can manufacture the outsourced components. Alternatively, this agent can find appropriate suppliers or outsourcing partner online via Internet or use the professional contacts and networking of the manufacturing firm. Three autonomous procedures are being followed to select appropriate partner. Firstly, it assesses certain criteria like availability, quality of raw material, cost, reliability and distance. Secondly, the technical expertise of partner is being evaluated in terms of sustaining engineering capability, design engineering capability etc. Finally, the financial and management aspects of partners are being considered. Partners are being reviewed by their credit history, balance sheet, credit worthiness and income statement.

3.4 Planning agent - It is responsible for planning and organization of all the tasks and operations being performed in manufacturing plant. This agent develops the manufacturing plan, which comprises of type of products to be manufactured, the associated quantities and the procedures followed for their production. Planning agent also decides the machines to be used for the production and the time slot. It works in close contact with maintenance agent. This assists in reducing the idle time for machines. Planning agent allocates the storage space for raw material and other products, accessories needed for the manufacturing. An appropriate storage space is chosen so as to minimize the logistics and storage costs. This agent establishes the smooth availability of raw materials in correct amount to the machines in manufacturing plant.

When the new order arrives, planning agent generates manufacturing plan using algorithm portfolio mechanism. Algorithm portfolio is composed of various algorithms such as Genetic Algorithm (GA), Tabu Search (TS), Simulated Annealing (SA), Bee Colony Optimization (BCO), Symbiotic Evolutionary Algorithm (SEA) and Ant Colony Optimization (ACO). In the literature, it was found that the selection of optimization 
algorithm depends on nature of problems and no particular optimization technique is appropriate for all problems. In the proposed approach, planning agent will execute algorithm portfolio and run various algorithms on different processors and link their performance to check their output on single as well as multiple processors. Its main goal is to select the algorithms that results in high performance in lieu of a particular problem i.e. to generate near optimal solution for a problem in designated time limits. This phenomenon results in increase in diverse problem solving capability and consistently high quality performance and reduction in costs associated with computation. A time limit will be allocated to it in generating the solution. Hence, the allocator will entrust an experimentation time to different algorithm agents and will conclude their priority order. The information received from each algorithm agent will be passed on to selector agent, which analyses this information and selects the algorithms to be bundled into portfolio to address the given problem. These algorithms are executed for quite a few iterations. In the end, the control agent will allocate improved knowledge to all algorithm agents. Therefore, instead of going for a single decision regarding the final selection of algorithm agent for leftover time period, the selector will return frequently to its choice again and again.

3.5 Knowledge base agent - It works in collaboration with rest of the agents like supplier selection agent, forecasting agent, designing agent, planning agent, maintenance agent, material planning agent and negotiation agent. All the information and decision made with respect to manufacturing processes and procedures is being stored in Knowledge base agent. It is accomplished by extracting information from all other agents and observing the tasks performed by them. This agent knows the current status of operations being executed for manufacturing a product. It is also aware of the sequence in which they were executed and the processing times associated with them. Knowledge base agent also does the record keeping of the successful and unsuccessful decisions taken by all agents. It assists in preventing those mistakes to occur again.

3.6 Forecasting agent - It calculates the quantity of raw material needed for manufacturing and sends this information to material planning agent, purchase order collection agent and supplier selection agent. It takes into account the current pattern of variations in demand happening in market. This agent is composed of very efficient and precise tools for forecasting and calculating the quantity and quality associated with recent trends in market. The main goal of this agent is to curtail the error in calculating the demand of clients keeping market swings in mind.

3.7 Designing Agent - This agent receives orders from customers with unique design formats made in software packages like CAD, CAM, etc. The received orders are being converted and communicated via Extensible Markup Language (XML) for the purpose of unified interaction and communication across the cloud and manufacturing infrastructure. Data format is being initially defined by interacting with cloud based definition database. These inferential data format definitions are in turn sent to software application bin having database of numerous software applications. The software application engine transforms the different software applications designs as per protocol. The resultant files 
are then sent to product file database for the purpose of matching the resources and production capabilities. These files are stored in and retrieved from cluster of cloud databases. If the required resources and production capabilities are available for operation then the files developed in XML format with product data are compiled and formatted in sequence. These files are then sent to design agent again. This agent then interacts with planning agent to update about the resources and manufacturing capabilities in lieu of processing a particular customer order with distinct designs.

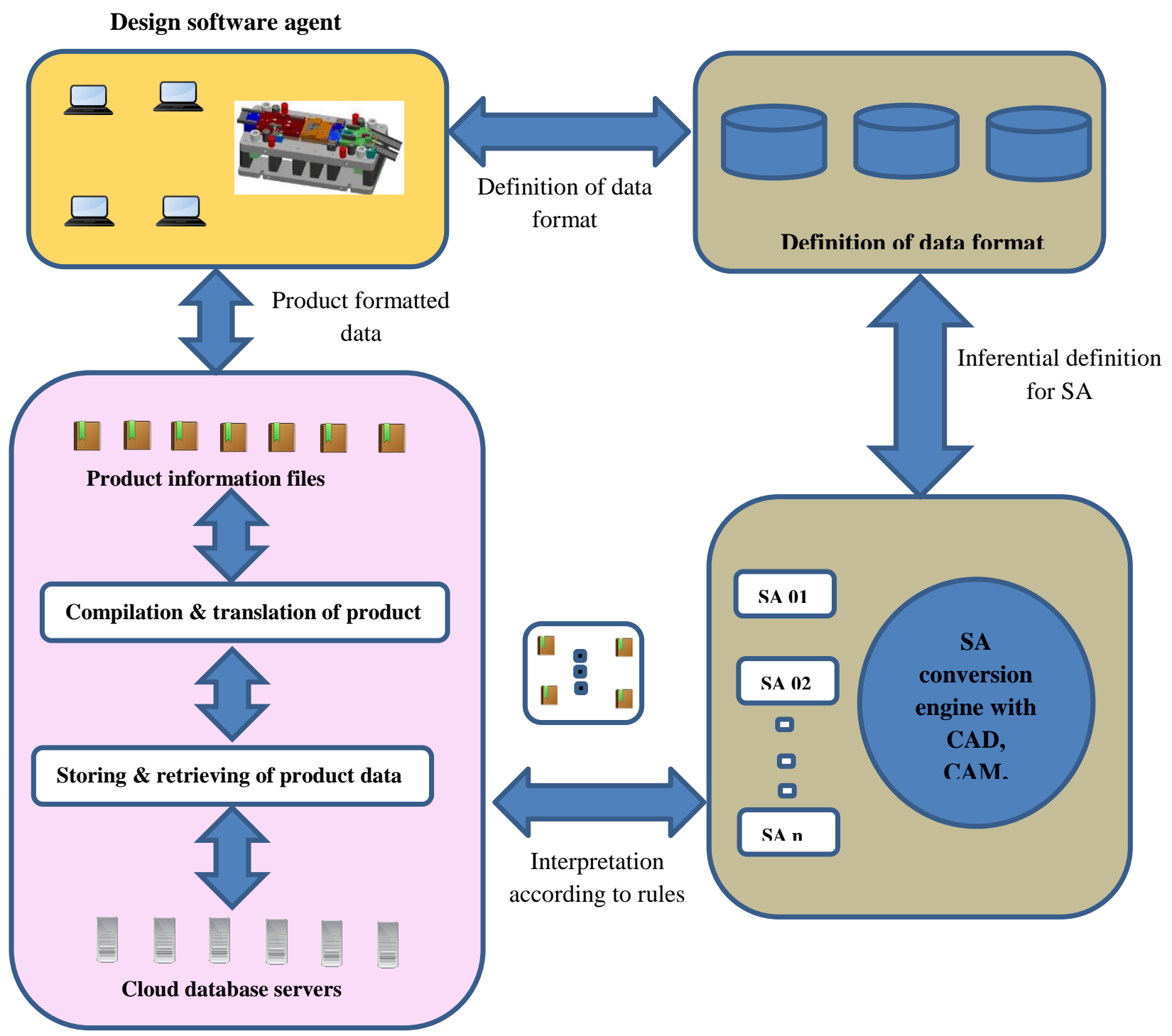

Figure 3 Schematic diagram of Design agent 
3.8 Maintenance agent - It keeps the track of machines in terms of their maintenance requirements, idle and in process stages, breakdown status, etc. by using the sensors in them. Maintenance agent decides the sequence in which various machines need repair and maintenance. This agent also takes a call if a machine has to be replaced or it can be repaired. The maintenance schedule will be developed so that there is minimum variance in the functioning of other agents. Replacing of machines is done after consultation with knowledge base agent. It also monitors the stock levels for materials needed for maintenance of machines. The maintenance agent is continuously in touch with planning agent. So, that planning agent is aware of which machine is going for maintenance and for what time period. In the case of disruptions, such as random machine breakdown, planning agent will contact maintenance agent and will generate efficient reschedule manufacturing plan.

3.9 Administrative agent - It monitors all the operations performed by other agents and ensure that they are legitimate. This agent particularly looks after guarantees, warranties, agreements, health and safety measures, transport security, etc. Administrative agent also manages all the financial accounts like payment to supplier, salaries of employees, customer's payments and miscellaneous transactions.

3.10 Transport Agent - It becomes active when it receives signal from purchase order collection agent regarding the transport of goods to a particular client. This agent makes the selection of mode of transport to be used for transporting the manufactured products. The transport mode chosen is an optimized trade-off between cheapest and quickest mode of transport. It also does record keeping of transport means used in the past. It informs knowledge base agent about its final decision by sending a signal.

3.11 Negotiation agent- The demand of customers of a manufacturing firm is fluctuating in nature both in terms of quantity and due date of delivery of their orders. If a new order is arrived or the existing client changes the due date. The planning agent performs the calculation if manufacturing facility is available then changes can be made easily. However, if after looking at due date, existing manufacturing capacity (number of machines available) and the current workload, this order cannot be accommodated. Then, purchase order collection agent tries to negotiate with the client. If the client is strict about the deadline then this issue is being referred to negotiation agent and the extent of price reduction will be informed. Initially, negotiation agent analyses the past record of this client. If the client is a very reliable business partner and the short term and long term prospects of the manufacturing firm are at stake. Then, negotiation agent collects the information of existing order being processed from planning agent and the existing 
manufacturing plan. The planning agent will perform the calculation using algorithm portfolio and generate all possible combinations such as list of clients with whom negotiation process has to be done, corresponding level of minimum due date extension and the extent of price reduction. This criterion is developed in a way so that manufacturing firm is making profit above a threshold level by accommodating a certain client's order and giving price reduction to other orders. Based on the collected information, the negotiation agent will start negotiating with clients. The negotiation process is complex. In literature, various metaheuristic algorithms have been developed for this purpose. However, no particular algorithm is suitable for all scenarios. In the proposed architecture, negotiation agent will use algorithm portfolio method as described in section 3.4 for negotiation. The negotiation agent will initiate the process over the cloud. The moment the process is started, listed clients will receive a notification. The interested clients will $\log$ in to the cloud using SaaS and participate in the bidding process. All participating clients will engage in the negotiation process real time. They will submit their initial bid (new price and due date). The artificial intelligence present on the cloud will perform the calculation using algorithm portfolio. It will inform those clients whose bid is matching the criteria of negotiation agent. If the information submitted by the clients does not meet the criteria then it will provide another opportunity to all clients for further bidding. This process will continue until their outcome matches the specifications of the criteria. Once a state of mutual agreement between a client or a set of clients and negotiation agent is being achieved then purchase order collection agent and planning agent are informed about it. Purchase order collection agent has informed the new client that their order has been accepted. The planning agent liaises with negotiation agent and new manufacturing plan is developed.

3.12 Communication Ontology- In this multi agent framework, one agent collaborates with other agent via sending 'help' signals. Agents lying in preceptor range of sender agent can only respond to this signal. A 'help' signal $H_{i}^{t}$ is sent by ith agent regarding cooperative help in task $t$ can be expressed mathematically as:

$H_{i}^{t}=\left(a_{i}, t_{t}\right) \quad \forall A^{P R_{i}}$

$A^{P R_{i}}$ denotes the collection of agents lying in the perceptory range of agent (PR) of agent i and can be written as :

$A^{P R_{i}}=\left\{a_{j} \forall \frac{a}{a_{j} P R_{i}}\right\}$

$\in A^{P R_{i}} \quad \exists$ a,$A^{P R_{i}} \in \wedge\left(a_{i}\right)$

$a_{j}{ }^{P R_{i}}$ denotes the $\mathrm{j}$ agent within the perceptory range of agent $\mathrm{i}$ and can be written as :

$a_{j}^{P R}<\neg d_{i j} \mid<P R_{i}$ 
$\left|d_{i j}\right|$ denotes the distance between agent $\mathrm{i}$ and $\mathrm{j}$. In this study, Manhattan distance has been considered as compared to Euclidean distance because of its precision in parallel computing circumstances (Kumar and Mishra, 2011). After receiving a help signal from agent $\mathrm{i}$, agent $\mathrm{j}$ sends a reply signal $r_{j}^{i}$ which can be written as:

$$
r_{j}^{i}=\left(a_{j}, a_{i}, H_{i}^{t}\right)
$$

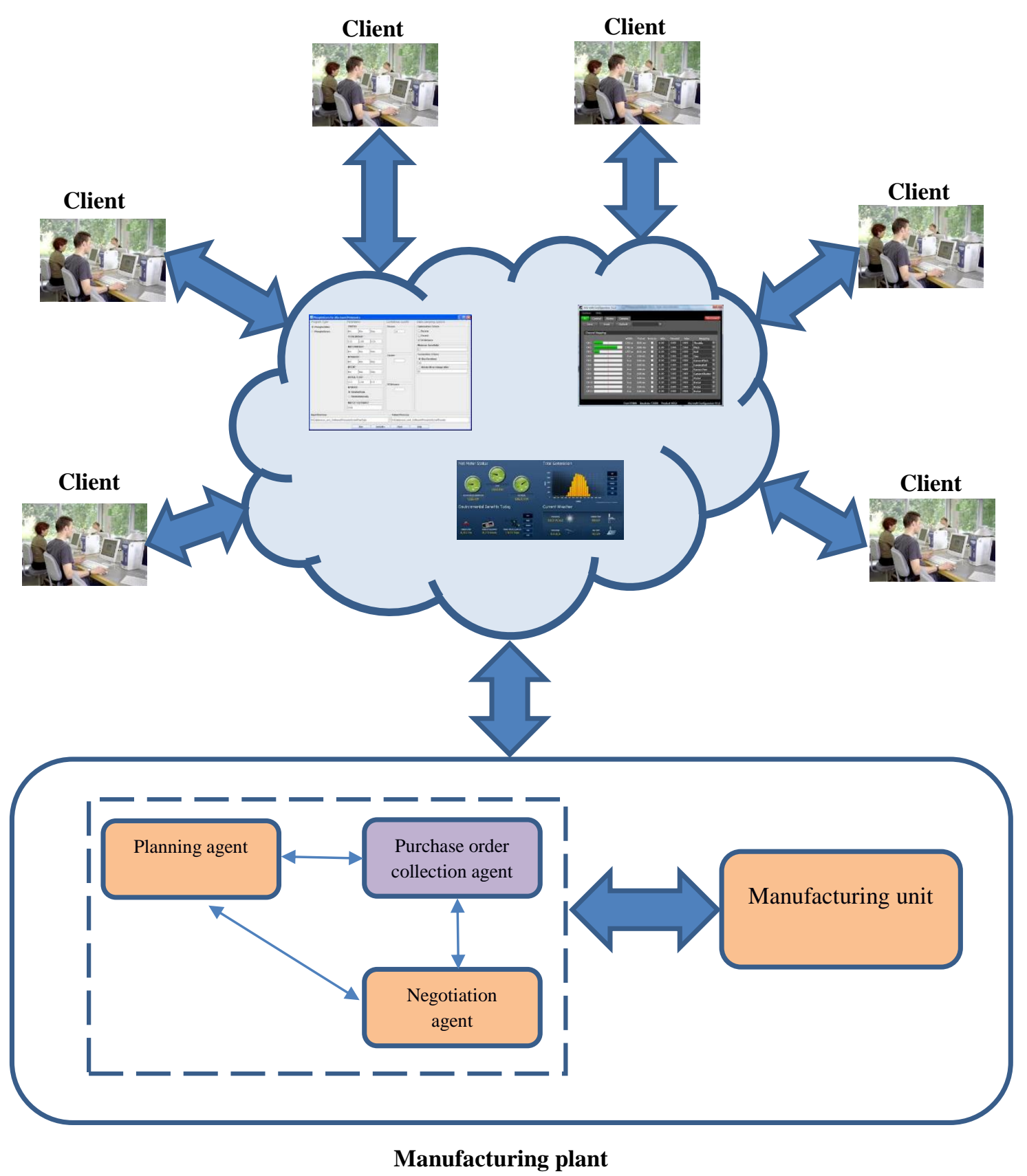

Figure 4 Highlighting operations of negotiation agent 
3.13 Communication channel - The accuracy of communication between agents defines the performance of this multi agent framework. The task of communication channel becomes strenuous in distributed manufacturing scenario. The agents interact among themselves via sending signals. The moment, they receive signal, an appropriate actions is taken by them. Literature consists of plenty of communication languages for precise communication like Agent Communication Language, KQML (Knowledge Query Manipulation Language), etc. MALLET (Multi Agent Logic Language for Encoding Teamwork) is one of renowned and popular communication language. It utilizes team oriented programming for the communication. This language assists in accurate encoding of information and smooth information flow in the system by deploying sequential and iterative processes, which could be either declarative or procedural. In this study, for precise and effective communication, agents will utilize MALLET communication channel. CAST (Collaborative Agents for Simulating Teamwork) is an interpreter for MALLET. It has been depicted in figure 5.

Agents define their functions, work plans and capabilities before starting their communication. They engage their tasks along with remaining agents depending on their condition. The latter agent replies by sending it pre requirements like information needed and knowledge to the former agent. The agents can communicate in different ways like Sequnetial (SEQ), Parallel (PAR), Choice Specific (CHOICE), Iterative (WHILE, FOR) or Conditional (IF). The agents initiate its operation after its pre requirements are fulfilled. If in a rare case, a particular agent is not able to meet its pre requirements then other agents rescue it by providing assistance.

3.14 Distributed Manufacturing communication channel- The agents located in a particular manufacturing plant will utilise the MALLET as described in section 3.13. However, there is a need for communication between different manufacturing units located in various geographical locations. The distributed manufacturing communication channel is being utilised for this purpose. It provides connectivity to all the agents and resources distributed across the globe. XML is used for these services. XML data structure developed by W3C standard. They can be conveniently translated into different web protocols. It has been depicted in diagram. Customers who are at vast geographical distances utilise this service cloud. They will exchange various required information via manufacturing service cloud. For example, design and product specifications of different parts manufactured at different geographical locations can be transformed into unified protocol and share the files in XML format over the cloud. 


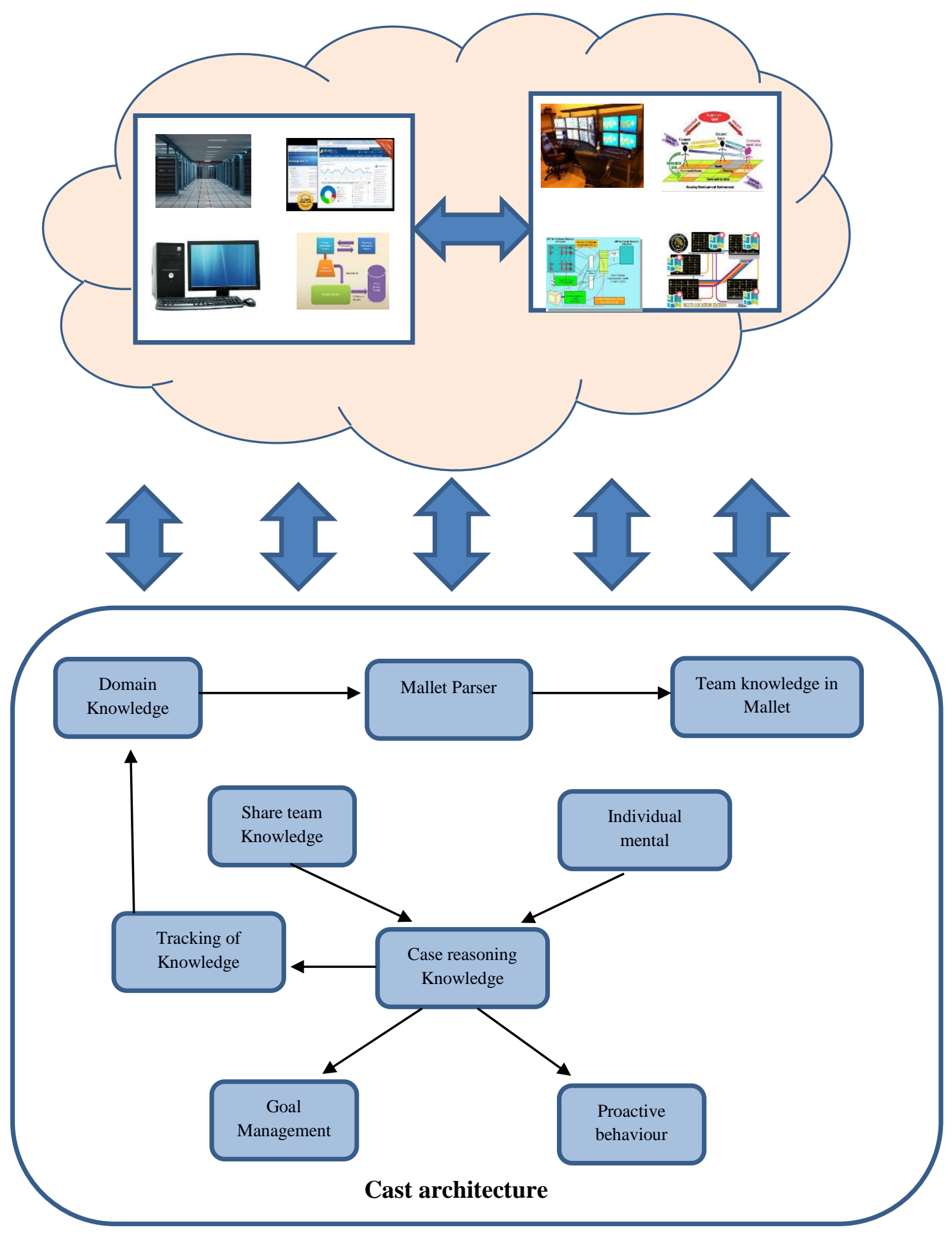

Figure 5 CAST architecture for cloud based multi agent framework 


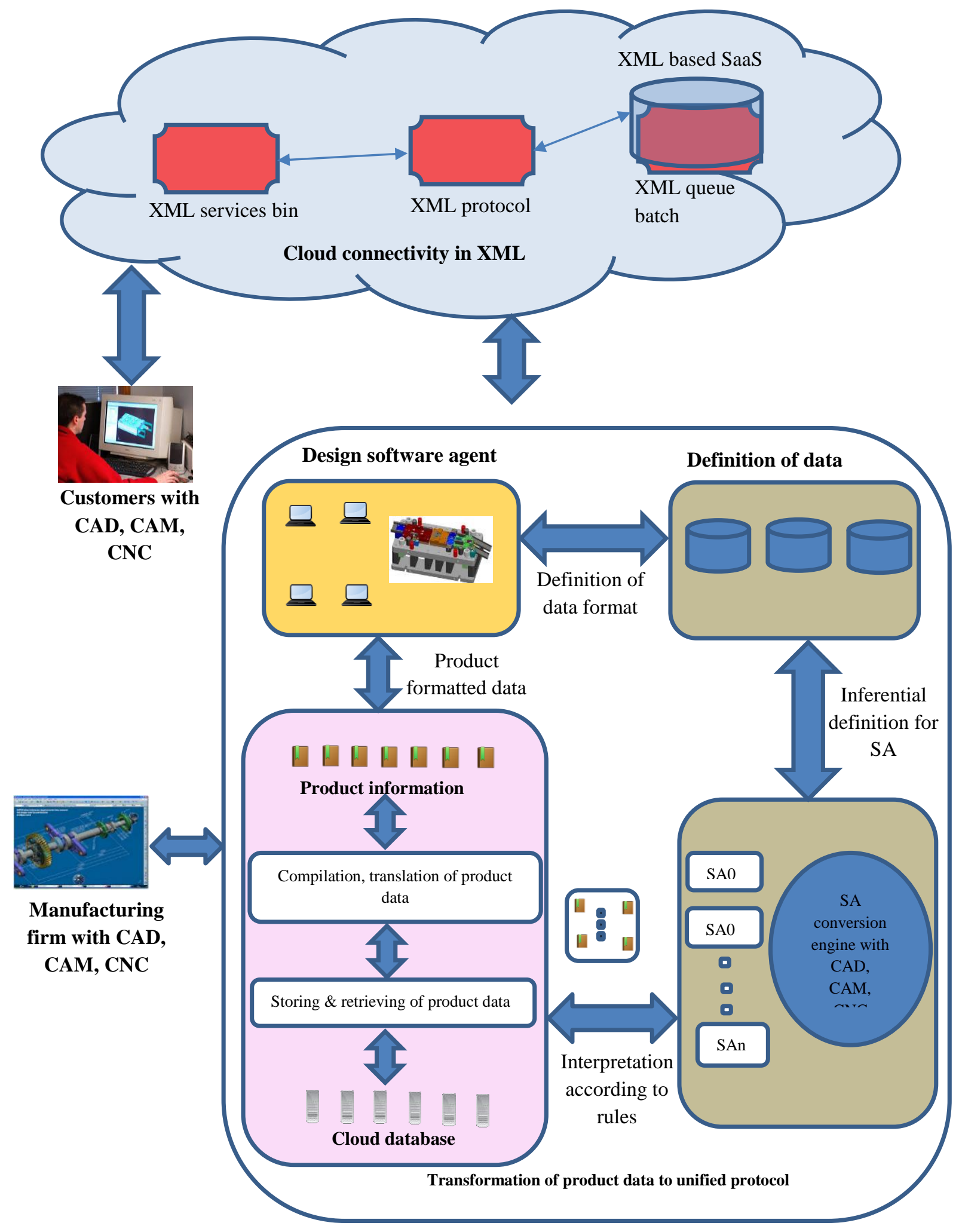

Figure 6 Schematic diagram of distributed manufacturing framework 


\section{Case Study}

This case study comprises of medium scale manufacturing company making various products. It is capable of making multiple products at a time and is utilizing cloud based multi agent system for improving their efficiency. Initially, purchase order collection agent has received fifteen orders from six different clients along with product details. Thereafter, purchase order collection agent interacted with planning agent to determine the due date of delivery of these products. The details of these orders and their respective quantities, operations involved and their precedence relationship is given in Table 1. Purchase order collection agent now decides the mode of payment and mode of delivery in lieu of these products by collaborating with administrative agent and transport agent respectively. Now, material-planning agent calculates the quantities of various raw materials needed. If the required amount of raw material is not available, then it will contact supplier selection agent, which will look for appropriate supplier by using its own procedures and database of knowledge base agent. Then, it will select the most appropriate supplier and give the order of raw materials needed for manufacturing. When the required raw materials are available in sufficient quantity, planning agent will come into action and assess the availability of machines with respect to the order received. It also takes the input of maintenance agent regarding the machines needing maintenance in near future. Based on received information, planning agent executes algorithm portfolio to generate manufacturing plan and run various algorithms on different processors and link their performance to check output on single as well as multiple processors. During the experiment, performance of genetic algorithm was better compared to other algorithms. The manufacturing plan generated by genetic algorithm is shown in Table 2 and is being passed on to manufacturing unit to manufacture the products. At this moment, the manufacturing process starts in the plant. After 10 days, client $\mathrm{C} 1$ has contacted purchase order collection agent and request them that they need 45 more quantities of the same product. This client is a very reliable customer of manufacturing firm. The manufacturing firm makes more profit from the order of this client as compared to other clients. Hence, both its short and long terms benefits will be accomplished if it is able to accommodate this increased order from the reliable client. Purchase order collection agent contacts planning agent and asks if this order can be accepted and delivered in the requested date by not affecting the order of other clients. Planning agent after analysing its manufacturing capability and the increased order, replied that it is not possible to process this order in the given due date. Purchase order collection agent approached negotiation agent and inform it about the extent of subsidised prices it can offer to other clients. The negotiation agent will take the information from planning agent about the current manufacturing plan and the list of clients and different options to accommodate the increased order of client $\mathrm{C} 1$. Planning agent will then generate the negotiation plan (extended due date of different combinations of clients and corresponding reduced price) in coordination with purchase order collection agent. Then, negotiation agent will contact these clients via cloud computing technology and starts negotiating with them accordingly. The other clients are happy with the fact that they are getting their products at fairly cheaper cost. As soon as client enter the 
details of their bid, the artificial intelligence present on the cloud will execute the negotiation using algorithm portfolio. During the negotiation, bids of client C3 have been accepted as they involve minimum changes in existing manufacturing plan as shown in Table 3. Negotiation agent will inform purchase order collection agent about it. Purchase order collection agent will inform the client $\mathrm{C} 1$ that its increased order has been accepted. It will also inform planning agent about the same along with the new combination of due dates of client C3. Now, planning agent will assess the manufacturing finished till date, previous manufacturing plan, availability of machines, inputs from maintenance agent. Then, it executes algorithm portfolio to develop a new manufacturing plan to process orders of all the clients within their respective due dates of delivery. In the experiment, performance of simulated annealing was better compared to other algorithms used in algorithm portfolio. The revised manufacturing plan generated by algorithm portfolio method is shown in table 4 . Finally, manufacturing starts in the plant as per the newly developed manufacturing plan. This cloud based multi agent architecture has helped the manufacturing firm to accommodate unprecedented orders in order to boost their revenue and help them in achieving their short and long-term benefits.

Table 1. Details of customer orders

\begin{tabular}{|c|c|c|c|c|c|c|c|}
\hline $\begin{array}{c}\text { Serial } \\
\text { Number }\end{array}$ & Client & Orders & Operation sequence & $\begin{array}{l}\text { Price } \\
\text { per } \\
\text { item }\end{array}$ & Quantity & $\begin{array}{c}\text { Operations } \\
\text { involved }\end{array}$ & $\begin{array}{c}\text { Delivery } \\
\text { time (days) }\end{array}$ \\
\hline 1 & \multirow[t]{2}{*}{$\mathrm{C} 1$} & P1 & $1 \rightarrow 2->3$ & $£ 100$ & 45 & $1,2,3$ & \multirow[t]{2}{*}{27} \\
\hline 2 & & $\mathrm{P} 2$ & $4->5->6$ & $£ 120$ & 45 & $4,5,6$ & \\
\hline 3 & \multirow{3}{*}{$\mathrm{C} 2$} & P3 & $7->8$ & $£ 70$ & 30 & 7,8 & \multirow[t]{3}{*}{28} \\
\hline 4 & & $\mathrm{P} 4$ & $9->10->11$ & $£ 75$ & 25 & $9,10,11$ & \\
\hline 5 & & $\mathrm{P} 5$ & $12->13->14$ & $£ 90$ & 40 & $12,13,14$ & \\
\hline 6 & \multirow[t]{2}{*}{$\mathrm{C3}$} & P6 & $15->16->17$ & $£ 50$ & 23 & $15,16,17$ & \multirow[t]{2}{*}{31} \\
\hline 7 & & P7 & $18->19->20$ & $£ 70$ & 20 & $18,19,20$ & \\
\hline 8 & \multirow{3}{*}{ C4 } & P8 & 21 & $£ 65$ & 25 & 21 & \multirow[t]{3}{*}{33} \\
\hline 9 & & P9 & $22->23->24$ & $£ 95$ & 30 & $22,23,24$ & \\
\hline 10 & & P10 & $25->26->27$ & $£ 80$ & 30 & $25,26,27$ & \\
\hline 11 & \multirow[t]{3}{*}{$\mathrm{C} 5$} & P11 & $28->29->30$ & $£ 50$ & 20 & $28,29,30$ & \multirow[t]{3}{*}{35} \\
\hline 12 & & P12 & $31->32$ & $£ 60$ & 20 & 31,31 & \\
\hline 13 & & P13 & $33->34->35$ & $£ 60$ & 20 & $33,34,35$ & \\
\hline 14 & \multirow[t]{2}{*}{$\mathrm{C} 6$} & P14 & $36->37$ & $£ 70$ & 30 & 36,37 & \multirow[t]{2}{*}{20} \\
\hline 15 & & P15 & $38->39->40$ & $£ 95$ & 20 & 38,3940 & \\
\hline
\end{tabular}


Table 2. Initial sequence generated by planning agent

\begin{tabular}{|c|c|c|c|c|c|c|c|c|c|c|}
\hline \multirow[t]{2}{*}{ M1 } & \multicolumn{2}{|c|}{ Operation 38} & \multicolumn{2}{|c|}{ Operation 16} & \multicolumn{2}{|c|}{ Operation 40} & \multicolumn{2}{|c|}{ Operation 34} & \multicolumn{2}{|c|}{ Operation 27} \\
\hline & 0 & 5 & 9 & 15 & 15 & 20 & 20 & 25 & 28 & 33 \\
\hline \multirow[t]{2}{*}{ M2 } & \multicolumn{2}{|c|}{ Operation 21} & \multicolumn{2}{|c|}{ Operation 33} & \multicolumn{2}{|c|}{ Operation 24} & \multicolumn{2}{|c|}{ Operation 20} & & \\
\hline & 0 & 6 & 6 & 11 & 12 & 16 & 27 & 31 & & \\
\hline \multirow[t]{2}{*}{ M3 } & \multicolumn{2}{|c|}{ Operation 4} & \multicolumn{2}{|c|}{ Operation 15} & \multicolumn{2}{|c|}{ Operation 32} & \multicolumn{2}{|c|}{ Operation 37} & \multicolumn{2}{|c|}{ Operation 10} \\
\hline & 0 & 5 & 5 & 9 & 9 & 13 & 13 & 19 & 19 & 24 \\
\hline \multirow{2}{*}{ M4 } & \multicolumn{2}{|c|}{ Operation 12} & \multicolumn{2}{|c|}{ Operation 14} & \multicolumn{2}{|c|}{ Operation 5} & & & & \\
\hline & 0 & 6 & 11 & 16 & 16 & 23 & & & & \\
\hline \multirow[t]{2}{*}{ M5 } & \multicolumn{2}{|c|}{ Operation 2} & \multicolumn{2}{|c|}{ Operation 18} & & & & & & \\
\hline & 7 & 14 & 14 & 21 & & & & & & \\
\hline \multirow{2}{*}{ M6 } & \multicolumn{2}{|c|}{ Operation 1} & \multicolumn{2}{|c|}{ Operation 35} & & & & & & \\
\hline & 0 & 7 & 30 & 35 & & & & & & \\
\hline \multirow[t]{2}{*}{ M7 } & \multicolumn{2}{|c|}{ Operation 28} & \multicolumn{2}{|c|}{ Operation 7} & \multicolumn{2}{|c|}{ Operation 9} & Op & n 3 & Op & \\
\hline & 0 & 5 & 5 & 10 & 10 & 14 & 14 & 19 & 21 & 27 \\
\hline M8 & & n 23 & & & & & & & & \\
\hline & 7 & 12 & & & & & & & & \\
\hline M9 & & n 31 & & on 36 & & n 8 & Op & n 25 & Op & on 26 \\
\hline & 0 & 6 & 6 & 10 & 10 & 16 & 16 & 22 & 22 & 28 \\
\hline M10 & & n 13 & & on 6 & & & & & & \\
\hline & 6 & 11 & 22 & 27 & & & & & & \\
\hline M11 & & n 39 & & on 29 & & n 17 & & & & \\
\hline & 5 & 12 & 12 & 19 & 19 & 24 & & & & \\
\hline & & & & & & & & & & \\
\hline M12 & & on 22 & & on 30 & & n 11 & & & & \\
\hline & 0 & 7 & 19 & 24 & 24 & 28 & & & & \\
\hline
\end{tabular}


Table 3. Details of customer orders after negotiation

\begin{tabular}{|c|c|c|c|c|c|c|c|}
\hline $\begin{array}{c}\text { Serial } \\
\text { Number }\end{array}$ & Client & Orders & Operation sequence & $\begin{array}{l}\text { Price } \\
\text { per } \\
\text { item }\end{array}$ & Quantity & $\begin{array}{c}\text { Operations } \\
\text { involved }\end{array}$ & $\begin{array}{c}\text { Delivery } \\
\text { time (days) }\end{array}$ \\
\hline 1 & \multirow[t]{2}{*}{$\mathrm{C} 1$} & P1 & $1 \rightarrow 2->3$ & $£ 100$ & 45 & $1,2,3$ & \multirow[t]{2}{*}{35} \\
\hline 2 & & $\mathrm{P} 2$ & $4->5->6$ & $£ 120$ & 45 & $4,5,6$ & \\
\hline 3 & \multirow{3}{*}{$\mathrm{C} 2$} & P3 & $7 \rightarrow 8$ & $£ 70$ & 30 & 7,8 & \multirow[t]{3}{*}{28} \\
\hline 4 & & $\mathrm{P} 4$ & 9 -> 10 -> 11 & $£ 75$ & 25 & $9,10,11$ & \\
\hline 5 & & P5 & $12->13->14$ & $£ 90$ & 40 & $12,13,14$ & \\
\hline 6 & \multirow[t]{2}{*}{$\mathrm{C3}$} & P6 & $15 \rightarrow 16->17$ & $£ 45$ & 23 & $15,16,17$ & \multirow[t]{2}{*}{41} \\
\hline 7 & & P7 & $18->19->20$ & $£ 63$ & 20 & $18,19,20$ & \\
\hline 8 & \multirow{3}{*}{$\mathrm{C} 4$} & P8 & 21 & $£ 65$ & 25 & 21 & \multirow[t]{3}{*}{33} \\
\hline 9 & & P9 & $22->23->24$ & $£ 95$ & 30 & $22,23,24$ & \\
\hline 10 & & P10 & $25->26->27$ & $£ 80$ & 30 & $25,26,27$ & \\
\hline 11 & \multirow[t]{3}{*}{$\mathrm{C} 5$} & P11 & $28->29->30$ & $£ 50$ & 20 & $28,29,30$ & \multirow[t]{3}{*}{35} \\
\hline 12 & & P12 & $31->32$ & $£ 60$ & 20 & 31,31 & \\
\hline 13 & & P13 & $33->34->35$ & $£ 60$ & 20 & $33,34,35$ & \\
\hline 14 & \multirow[t]{2}{*}{ C6 } & P14 & $36->37$ & $£ 70$ & 30 & 36,37 & \multirow[t]{2}{*}{20} \\
\hline 15 & & P15 & $38->39$-> 40 & $£ 95$ & 20 & 38,3940 & \\
\hline
\end{tabular}


Table 4. Operation sequence after negotiation

\begin{tabular}{|c|c|c|c|c|c|c|c|c|c|c|c|c|}
\hline \multirow[t]{2}{*}{ M1 } & \multicolumn{2}{|c|}{ Operation 38} & \multicolumn{2}{|c|}{ Operation 16} & \multicolumn{2}{|c|}{ Operation 40} & \multicolumn{2}{|c|}{ Operation 34} & \multicolumn{2}{|c|}{ Operation 27} & & \\
\hline & 0 & 5 & 9 & 15 & 15 & 20 & 20 & 25 & 28 & 33 & & \\
\hline \multirow[t]{2}{*}{ M2 } & \multicolumn{2}{|c|}{ Operation 21} & \multicolumn{2}{|c|}{ Operation 33} & \multicolumn{2}{|c|}{ Operation 24} & \multicolumn{2}{|c|}{ Operation 20} & & & & \\
\hline & 0 & 6 & 6 & 11 & 12 & 16 & 37 & 41 & & & & \\
\hline \multirow[t]{2}{*}{ M3 } & \multicolumn{2}{|c|}{ Operation 4} & \multicolumn{2}{|c|}{ Operation 15} & \multicolumn{2}{|c|}{ Operation 32} & \multicolumn{2}{|c|}{ Operation 37} & \multicolumn{2}{|c|}{ Operation 10} & & \\
\hline & 0 & 5 & 5 & 9 & 9 & 13 & 13 & 19 & 19 & 24 & & \\
\hline \multirow[t]{2}{*}{ M4 } & \multicolumn{2}{|c|}{ Operation 12} & \multicolumn{2}{|c|}{ Operation 14} & \multicolumn{2}{|c|}{ Operation 5} & \multicolumn{2}{|c|}{ Operation 5} & & & & \\
\hline & 0 & 6 & 11 & 16 & 16 & 23 & 23 & 30 & & & & \\
\hline \multirow{2}{*}{ M5 } & \multicolumn{2}{|c|}{ Operation 2} & \multicolumn{2}{|c|}{ Operation 2} & \multicolumn{2}{|c|}{ Operation 18} & & & & & & \\
\hline & 7 & 14 & 18 & 25 & 25 & 32 & & & & & & \\
\hline \multirow{2}{*}{ M6 } & \multirow{2}{*}{\multicolumn{2}{|c|}{ Operation 1}} & \multirow{2}{*}{\multicolumn{2}{|c|}{ Operation 1}} & \multirow{2}{*}{\multicolumn{2}{|c|}{ Operation 35}} & & & & & & \\
\hline & & & & & & & & & & & & \\
\hline & & & & & & & & & & & & \\
\hline M7 & Ope & n 28 & Op & on 7 & Ope & on 9 & Ope & on 3 & $\mathrm{Op}$ & on 3 & & on 19 \\
\hline & 0 & 5 & 5 & 10 & 10 & 14 & 14 & 19 & 26 & 31 & 32 & 38 \\
\hline M8 & Ope & n 23 & & & & & & & & & & \\
\hline & 7 & 12 & & & & & & & & & & \\
\hline M9 & Ope & n 31 & Ope & n 36 & Ope & n 8 & Oper & n 25 & Ope & n 26 & & \\
\hline & 0 & 6 & 6 & 10 & 10 & 16 & 16 & 22 & 22 & 28 & & \\
\hline M10 & Ope & n 13 & $\mathrm{Op}$ & n 4 & Ope & n 6 & Ope & n 6 & & & & \\
\hline & 6 & 11 & 11 & 16 & 22 & 27 & 30 & 35 & & & & \\
\hline M11 & Ope & n 39 & Ope & n 29 & Oper & n 17 & & & & & & \\
\hline & 5 & 12 & 12 & 19 & 19 & 24 & & & & & & \\
\hline M12 & Ope & n 22 & Ope & n 30 & Oper & n 11 & & & & & & \\
\hline & 0 & 7 & 19 & 24 & 24 & 28 & & & & & & \\
\hline
\end{tabular}




\section{Conclusion}

Manufacturing systems are implementing sophisticated techniques to improve their responsiveness to cope with the fluctuating demand of their customers. They are following the principles of distributing manufacturing to address this issue of uncertainty in demand. The operations and decision making of a manufacturing firm gets affected by both internal factors like breakdown of machines and external factors like change in the existing order or a given due date for delivery of products. If these issues are not resolved efficiently, they can do serious damage to their current businesses and long-term prospects. Keeping these complications in mind, this study proposes a cloud based multi agent framework for distributed manufacturing. Eleven autonomous agents are being utilised to optimise all the operations of the manufacturing firm. The cloud computing technology integrates these autonomous agents, multiple clients, and various suppliers on a single platform for real time and rapid exchange of information. The agents employed in this framework are capable to deal with the internal and external interferences keeping in mind the short and long term prospects of the manufacturing firm. Planning and negotiation agents are utilising algorithm portfolio, which is a set of meta-heuristic techniques of operation management for effective planning and to deal with the internal and external disruptions efficiently. The algorithm portfolio helps these agents to choose the optimum combination of the meta-heuristic techniques to improve the efficiency and revenue of manufacturing firm. The mechanism of this study is being demonstrated in the case study section. This framework is flexible enough to be used on most of the manufacturing systems. In the future, additional meta-heuristic techniques could be added to the algorithm portfolio to make planning and negotiation agent more efficient in their decision-making. Further, additional parameters could be incorporated in the given framework to make the proposed model more potent in real world.

\section{Acknowledgement}

The authors would like to thank the project 'A cross country examination of supply chain barriers on market access for small and medium firms in India and UK' (Ref no: PM130233) funded by British Academy, UK for supporting this research.

\section{References}

Al-Hudhaif, S., Alkubeyyer, A., 2011. E-commerce adoption factors in Saudi Arabia. International Journal of Business Management. 6 (9), 122-133. 
Angela Lin, Nan-Chou Chen ., 2012. Cloud computing as an innovation: Perception, attitude, and adoption. International Journal of Information Management .32 (2012),533-540.

Bo, X., Zhiming W., 2003. Modelling of supply chain: a multi-agent approach. In 2003 American control conference. Denver, CO, United States.

Caridi, M., Cavalieri, S., 2004. Multi-agent systems in production planning and control: an overview. Production Planning and Control 15 (2), 106-118.

Chen, Z.L., 2010. Integrated production and outbound distribution scheduling: Review and extensions. Operations Research, 58(1), 130-148.

Chien, T. H., Lin, Y. I., \& Tien, K. W. (2013). Agent-based negotiation mechanism for multiproject human resource allocation. Journal of Industrial and Production Engineering, 30(8), 518-527.

Guo, Z. X., Wong, W. K., \& Guo, C. (2014). A cloud-based intelligent decision-making system for order tracking and allocation in apparel manufacturing. International Journal of Production Research, 52(4), 1100-1115.

Huang, C. J., \& Liao, L. M. (2012). A multi-agent-based negotiation approach for parallel machine scheduling with multi-objectives in an electro-etching process. International Journal of Production Research, 50(20), 5719-5733.

Huang, J.-Y., \& Yao, M.-J., 2013. On the optimal lot-sizing and scheduling problem in serialtype supply chain system using a time-varying lot-sizing policy. International Journal of Production Research, 51 (3), 735-750

Jose Barbosa, Paulo Leitao, Emmanuel Adam and Damien Trentesaux., 2015. Dynamic self organization in holonic multi-agent manufacturing systens: The ADACOR evolution. Computers in Industry. 66(2015) 99-111.

Kim, H. J., Chiotellis, S., \& Seliger, G., 2009. Dynamic process planning control of hybrid disassembly systems. International Journal of Advanced Manufacturing Technology. 40(9-10), 1016-1023.

Kumar, V., \& Mishra, N. (2011). A multi-agent self correcting architecture for distributed manufacturing supply chain. Systems Journal, IEEE, 5(1), 6-15.

Li, X. Y., Shao, X. Y., Gao, L., \& Qian, W. R., 2010. An agent-based approach for integrated process planning and scheduling. Expert Systems with Applications. 37(2), 1256-1264.

Lim, M. K., Tan, K., \& Leung, S. C. (2013). Using a multi-agent system to optimise resource utilisation in multi-site manufacturing facilities. International Journal of Production Research, 51(9), 2620-2638.

Moyaux, T., Chaib-draa, B., 2006. Supply chain management and multi agent systems: an overview. In: Chaib-draa, B., Müller, J.P. (Eds.), Multi-agent-Based Supply Chain Management. Springer, USA. 
N .He, D.Z. Zhang and Q.Li., 2014. Agent based Hierarchical production planning and scheduling in make-to-order manufacturing system .International Journal of Production Economics. 149(2014) 117-130.

Parunak, H.V., 1999. Industrial and practical applications of DAI. In: Weiss, G. (Ed.), Multi Agent Systems-A Modern Approach to Distributed Modern Approach to Artificial Intelligence. The MIT Press, Cambridge, Massachusetts, London: England.

Renna, P. (2011). Multi-agent based scheduling in manufacturing cells in a dynamic environment. International Journal of Production Research, 49 (5), 1285-1301.

Singh, A., Mishra, N., Ali, S. I., Shukla, N., \& Shankar, R. (2015). Cloud computing technology: Reducing carbon footprint in beef supply chain. International Journal of Production Economics, 164, 462-471.

Swaminathan, J., Smith., S., Sadeh, N., 1998. Modelling supply chain dynamics: a multiagent approach. Decision Sciences. 29 (3), 607-632.

Wang, D., Yung, K.L., Ip, W.H., 2001. A heuristic genetic algorithm for subcontractor selection in a global manufacturing environment. IEEE Transactions on Systems. Man and Cybernetics 32 (2), 189-198.

Wang, L. H., Feng, H. Y., \& Cai, N. X., 2003. Architecture design for distributed process planning. Journal of Manufacturing Systems. 22(2), 99-115.

Wong, T. N., \& Fang, F. (2010). A multi-agent protocol for multilateral negotiations in supply chain management. International Journal of Production Research, 48(1), 271-299.

Yu, C., \& Wong, T. N. (2015). A multi-agent architecture for multi-product supplier selection in consideration of the synergy between products. International Journal of Production Research, (ahead-of-print), 1-24.

Yu, F., Kaihara, T., Fujii, N., Sun, C., \& Yang, W. (2015). A multi-attribute multi-item negotiation mechanism of supply chain networks between buyers and sellers. International Journal of Production Research, (ahead-of-print), 1-18. 
